\title{
Design and characterization of nanostructured SERS substrates based on gold nanostars
}

\author{
V.O. Yukhymchuk ${ }^{1}$, O.M. Hreshchuk ${ }^{1 *}$, M.Ya. Valakh ${ }^{1}$, Ye.G. Gule ${ }^{1}$, M.A. Skoryk ${ }^{2}$, V.S. Efanov ${ }^{1,2}$, \\ N.A. Matveevskaya ${ }^{3}$, T.G. Beynik ${ }^{3}$ \\ ${ }^{1}$ V. Lashkaryov Institute of Semiconductor Physics, NAS of Ukraine; \\ 45, prospect Nauky, 03680 Kyiv, Ukraine \\ ${ }^{2}$ Nanomedtech LLC, 68, Antonovych str., 03680 Kyiv, Ukraine \\ ${ }^{3}$ State Scientific Institution "Institute for Single Crystals", NAS of Ukraine \\ 60, prospect Nauky, 61178 Kharkiv, Ukraine \\ *E-mail:Hreshchuk@gmail.com
}

\begin{abstract}
Using the method of chemical synthesis, effective SERS substrates were developed, which were the films of gold nanoparticles possessing the form of stars. The peculiarity of star-shaped nanoparticles is that they could enhance the electric field of the radiating dipole not only by setting the frequency of the plasmon absorption band at the frequency of the exciting laser radiation, but additionally due to significant contribution to Raman scattering of the so-called "hot spots" that exist at the edges of the stars. "Nanostar"-based films were prepared using both direct deposition of gold nanoparticles from colloidal solution on the glass substrate and on the layer of polycation of polydiallyldimethyl ammonium bromide on the surface of glass substrates. Surface morphology of the obtained films was investigated using scanning electron microscopy. Plasmon absorption spectra were obtained using the method of optical absorption. Variation of geometrical parameters inherent to these nanostructures allows configuring the position of the plasmonic absorption band maximum close to the laser excitation wavelength. The coefficient of enhancement of Raman signal was evaluated for Rhodamine 6G molecules deposited on the designed SERS substrates (for different wavelengths of the exciting laser radiation, namely: $\lambda=633,532$ and $457 \mathrm{~nm}$ ).
\end{abstract}

Keywords: SERS, SEM, Raman scattering, absorption spectra, gold nanoparticles, "nanostars".

Manuscript received 03.11.16; revised version received 07.02.17; accepted for publication 01.03.17; published online 05.04.17.

\section{Introduction}

Surface-enhanced Raman spectroscopy (SERS) is an efficient analytical method that is intensively developing, and every year it finds more commonly usage in diagnostics of substances in chemistry, materials science, medicine, biology, pharmacology, ecology etc. [1-5]. SERS allows performing the analysis of substances available in low concentrations in solutions or deposited in the amount of one monolayer on nanostructured metal substrate. The SERS method is a non-destructive express method like typical Raman spectroscopy that allows identifying the component composition of substances, the features of their molecular structure and so on.

The theoretical and experimental studies show that the effect of SERS enhancement is based on two mechanisms: chemical and electromagnetic $[1,2]$. The first mechanism is associated with collective electron oscillations (surface plasmons) in metallic 
nanostructures that create an electromagnetic field near the surface, which enhances the Raman scattering from molecules or semiconductor nanostructures deposited on the SERS substrate. The second mechanism is associated with formation of chemical bonds between the studied molecules and metal nanostructures, which provides a resonant energy exchange between them under laser excitation. The efficiency of SERS effect depends on morphology of nanostructured metal surface [6], the parameters of the exciting laser radiation [7], the conditions of deposition and properties of the substance [8] as well as on the distance of the latter from the surface of SERS substrate [9], etc.

It's necessary to have substrates covered with films of nanostructured gold or silver for effective application of SERS substrates for routine laboratory usage. Enhancing "platforms" should be characterized by such parameters as stability in time, lateral uniformity, simple and low cost fabrication, and significant enhancement of the Raman signal for a wide range of substances (from chemical and biological to semiconductor nanoclusters).

It is known that using the methods of colloidal synthesis various nanostructures could be synthesized, which are suitable for SERS substrates, for instance: core-shell [10] nanostructures, nanoparticles (NPs) with different shapes: spherical, triangles, cubes, wires and others [11]. Nanostructures in the form of stars could be the new type of efficient substrates for the SERS diagnostics [12]. On the one hand, this geometrical form of nanostructures causes the concentration of electrical fields at the edges of nanostars (NSTs), moreover, it enhances the electric field of radiating dipole not only by fitting the frequency of plasmon absorption band to the frequency of the exciting laser radiation, but also due to the presence of the so-called "hot spots" in the radiating system. The latter one is a superposition of electrical fields created by few closely placed metal nanostructures. Typically, the hot spots are not evenly placed on the surface of SERS substrate, and the area covered by them does not exceed $1 \%$ of the total area [13]. However, in [13] it was shown that even such a low percent of hot spots could make its contribution to total SERS enhancement higher than $24 \%$. That is why it is so important for nanostructures of SERS substrates to form hot spots.

It should be noted that gold NSTs could be used as biomarkers for address delivery of drugs in the human body or in detection of malignant tumors, etc [14]. In [15], it was shown that the intensity of SERS signal from molecules adsorbed on the surface of metal nanoparticles is by 1-2 orders of magnitude higher than the intensity of quantum dots (QDs) radiation. Moreover, in contrast to QDs, the SERS based biomarkers does not have such properties as photobleaching and gradual self-quenching of emitted radiation.

The aim of this work was to develop a method of synthesis of nanostructures in the form of stars for efficient SERS substrates, to investigate their properties, establish the relationship between the morphology and plasmonic absorption band and to determine the coefficient of enhancement of the Raman signal from analyte deposited on them.

\section{The method of synthesis of nanostructures}

The following chemical components were used for synthesis of gold NSTs and forming of films based on them: gold hydrochloric acid $\mathrm{HAuCl}_{4} \cdot 3 \mathrm{H}_{2} \mathrm{O} \geq 99.9 \%$, sodium citrate $\mathrm{Na}_{3} \mathrm{C}_{6} \mathrm{H}_{5} \mathrm{O}_{7} \geq 98 \%$, hydrochloric acid $\mathrm{HCl} \geq 99 \%$, silver nitrate $\mathrm{AgNO}_{3} \geq 99.8 \%$, ascorbic acid $\mathrm{C}_{6} \mathrm{H}_{8} \mathrm{O}_{6} \geq 99 \%$, 3-aminopropyl-triethoxysilane (APTES) $\mathrm{C}_{9} \mathrm{H}_{23} \mathrm{NO}_{3} \mathrm{Si} \geq 98 \%$, sulfuric acid $\mathrm{H}_{2} \mathrm{SO}_{4} \geq 98 \%$, hydrogen peroxide $\mathrm{H}_{2} \mathrm{O}_{2} \geq 30 \%$, poly (dyallildymetyl) ammonium bromide (PDADMAD) $\left(\mathrm{C}_{8} \mathrm{H}_{16} \mathrm{ClN}\right)_{\mathrm{n}}(20 \%$ aqueous solution) without further purification.

The two-stage method of chemical synthesis was chosen to obtain aqueous solutions with colloidal gold NSTs, which allowed to control the size and shape of the obtained nanoparticles (NPs). At the first stage of synthesis, the spherical gold NPs were obtained with an average size close to $12 \mathrm{~nm}$. The solution of spherical gold NPs was obtained by addition of $1.5 \mathrm{ml}$ of $1 \%$ solution of sodium citrate to $10 \mathrm{ml}$ of $0.001 \mathrm{M}$ of boiling solution of $\mathrm{HAuCl}_{4}$ and stirring. The solution was boiled for 15 min maintaining a constant volume, and then this solution was cooled in air. The obtained spherical gold NPs served as seeds for controlled growing the bulk nanostructures in the form of stars.

Gold cones with sharp peaks (the edges of the stars) were grown during the second stage of synthesis on the surface of the spherical gold NPs. This process was held due to the recovery of gold hydrochloric acid by ascorbic acid on the surface of Au NPs, in the presence of silver ions in solution. In order to obtain the gold nanostars, $0.1 \mathrm{ml}$ of the spherical $\mathrm{Au}$ seeds was added to $10 \mathrm{ml}$ of $2.5 \cdot 10^{-4} \mathrm{M}$ of $\mathrm{HAuCl}_{4}$ solution at $\mathrm{pH}=3$, the solution being under intense stirring. Then $0.002 \mathrm{ml}$ of $0.05 \mathrm{M}$ of $\mathrm{AgNO}_{3}$ solution and $0.5 \mathrm{ml}$ of $0.01 \mathrm{M}$ solution of ascorbic acid were quickly added. After adding ascorbic acid, the color of the solution drastically changed to blue, indicating the formation of Au NSTs.

The presence of silver ions in the solution was a prerequisite for formation of gold NPs of non-spherical shape, namely: creation and growth of sharp edges in NSTs along certain crystallographic facets of gold NPs. Silver is adsorbed on the facets of the gold NPs with the largest surface energy, thus forming there monolayers or submonolayers of $\mathrm{Ag}$ [16]. This leads to selective stabilization of the facets of $\{110\},\{310\}$ and $\{720\}$ of the gold NPs [17] and prevents the further growth of $\mathrm{Au}$ along these facets. Accordingly, further growth of gold NPs is anisotropic, and the growth is on the facets that have a lower surface energy. In particular, after stabilization of the facets $\{110\}$ adsorption of Au occurs on the facets $\{111\}$, resulting in formation of gold in the form of cones along this direction.

Films with gold NSTs were obtained using the self- 
assembly method $[18,19]$ on the glass substrates with the size $1 \times 1 \mathrm{~cm}$. The substrates were kept in the solution of $\mathrm{H}_{2} \mathrm{SO}_{4}: \mathrm{H}_{2} \mathrm{O}_{2} \quad(10: 1)$ during 10 hours to remove adsorbed contaminants, then repeatedly washed with bidistilled water by using ultrasound. Surface modification of substrates was performed using the 5\% alcohol solution of APTES when boiling for 1.5 hours. The modified substrates were repeatedly washed with alcohol and water by using ultrasound to remove the excess of APTES.

Formation of the films with star-like nanostructures on the glass substrate was carried out in two ways. In order to obtain a multilayer film, the substrates were placed in colloidal solution of Au NSTs, and then in PDADMAB with the concentration $2 \mathrm{mg} / \mathrm{ml}$, $M \sim 100,000-200,000$. Substrates were kept in the solution of NSTs overnight at $20^{\circ} \mathrm{C}$, then rinsed with bidistilled water and placed in solution of polycations for $20 \mathrm{~min}$ (SERS substrate of type I). Another way to form SERS substrates was in direct deposition of the colloidal solution of the gold NSTs on the cleaned glass substrate by drop-drying method in air (SERS substrate of the II type).

\section{Experimental methods}

Morphology and structure of the SERS substrates with Au NPs, synthesized using the above mentioned methods, were examined with the scanning electron microscope (SEM) Tescan Mira 3 LMU, transmission electron microscope (TEM) TEM-125 with an accelerating voltage of $100 \mathrm{kV}$, by high resolution TEM Jeol JSM-820 equiped with X-ray microanalysis system LINK IN 10000. Samples for TEM measurements were prepared by the standard method, namely, by deposition of aqueous suspensions of NPs onto the copper substrates coated with a thin layer of carbon, followed by drying at room temperature.

The elemental composition of substrates with gold NPs were examined by X-ray photoelectron spectroscopy (XPS) MXPS XP (Omicron) equipped with hemispherical electrostatic analyzer, photoelectrons were excited by $\mathrm{Mg} \mathrm{K} \alpha$-radiation $(h \gamma=1253.6 \mathrm{eV})$. The energy scale was calibrated using the line S1s $\left(E_{b}=285 \mathrm{eV}\right)$. The resolution of spectrometer was $1.0 \mathrm{eV}$, accuracy of determining the binding energy was $0.2 \mathrm{eV}$. The composition of the samples was determined by the surface area ratio for the bands $\mathrm{C} 1 \mathrm{~s}, \mathrm{O} 1 \mathrm{~s}, \mathrm{Au} 4 \mathrm{f}$, $\mathrm{Si} 2 \mathrm{p}, \mathrm{Cl} 2 \mathrm{p}$, and N1s of valence electron shells with account of the sensitivity coefficients. The thickness of the analyzed layer was $\sim 5 \mathrm{~nm}$.

The absorption spectra of obtained nanostructures were recorded using the double-beam spectrophotometer UV-VIS-IR Shimadzu UV-3600. Raman studies were performed at room temperature by using a spectrometer with monochromator equipped with $\mathrm{CCD}$ camera of Andor company. Solid-state lasers with the wavelengths of 532 and $457 \mathrm{~nm}$ and $\mathrm{He}^{-\mathrm{Ne}^{+}}$-laser with the wavelength $632.8 \mathrm{~nm}$ were used to excite the Raman scattering. The solution of Rhodamine 6G (R6G) in bidistilled water was used as analyte with concentrations $10^{-3}$ and $10^{-5} \mathrm{M}$. The solution of R6G was deposited on substrates by dispenser in the same amount of substance $\left(5 \cdot 10^{-6} 1\right)$ at approximately the same area, and then dried in air to obtain the thin film of analyte molecules on the surface of SERS substrates. To prevent damage of the structure of the investigated molecules, Raman spectra were excited by laser radiation with a minimally sufficient power to ensure their registration. To verify the homogeneity of the SERS substrates several points on each sample were examined and the time of accumulation of the signal was the same for all measurements.

\section{Results and discussion}

Scanning (SEM) and transmission (TEM) electron microscopy were used to characterize morphology of the studied nanostructures. The results are shown in Fig. 1.

The figure shows that the synthesized nanostructures have the form of stars with sharp edges. The length of the edges (cones) ranges from 10 to $25 \mathrm{~nm}$, the thickness at their base is $\sim 10 \mathrm{~nm}$, and at the end of the tip it is $\leq 4 \mathrm{~nm}$. Nanostars have almost the same lateral dimensions $\leq 55 \mathrm{~nm}$, and their size dispersion is $13 \%$. SEM images (Fig. 1b, c, d) show that the emplacement of the NSTs is quite tight, which causes formation of the so-called hot spots, where the value of the electric fields is significantly larger than the average value of electric field on the surface of SERS substrate.

The characteristic reflections of corresponding planes of (111) (200) (220) (311) obtained by electronic microdiffraction are indicative of a crystalline and facecentered cubic structure of the obtained gold NPs in the form of stars (Fig. 2).

The elemental composition of obtained gold NSTs was investigated using X-ray photoelectron spectroscopy (XPS). The obtained spectra of Au4f and Ag3d shells from the surface of the samples are shown in Fig. 3. It was ascertained that the energy of the line $A u 4 f_{7 / 2}$ for all the samples equals to $84.0 \pm 0.2 \mathrm{eV}$, and it corresponds to the metallic state of the gold. Since the silver line doesn't have a pronounced chemical shift, namely, for the most of the silver compounds the binding energy of $\mathrm{Ag} 3 \mathrm{~d}$ does not differ more than by the value $0.2 \mathrm{eV}$ as compared to the binding energy of metallic silver $(368.1 \mathrm{eV})$ [17]. Therefore, it is impossible to unambiguously identify the chemical state of the silver from XPS spectra (Fig. 3). However, since there were not observed any lines from chlorine $\left(\mathrm{HAuCl}_{4}\right.$ and $\left.\mathrm{HCl}\right)$ in the spectra of the samples, and formation of silver oxide in the process of synthesis was impossible (from the chemical viewpoint), it could be assumed that silver could be found in the metallic state for all the samples with gold NSTs. 

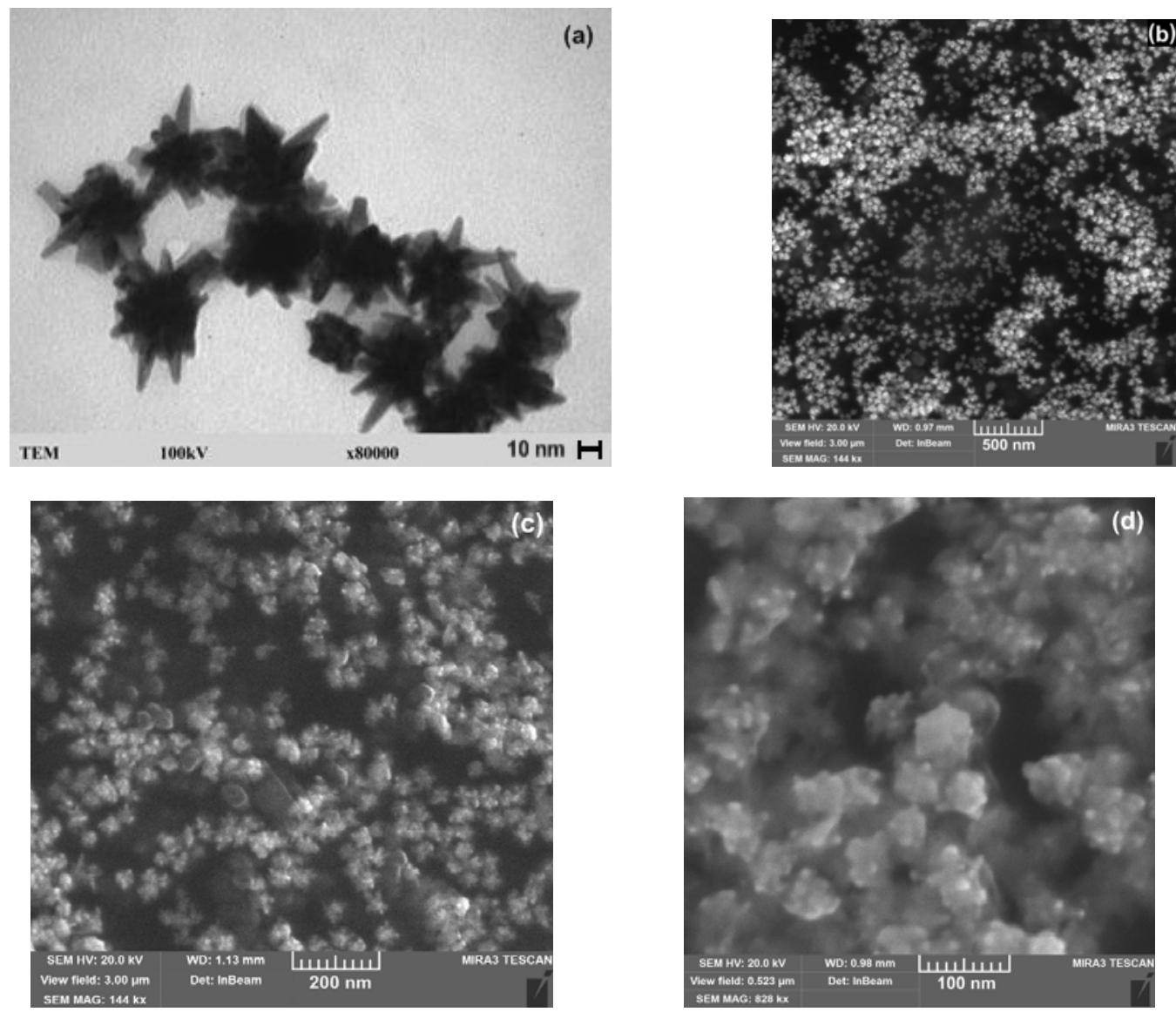

Fig. 1. TEM $(a)$ and SEM $(b, c, d)$ images of the gold nanostructures in the form of bulk "stars".

As noted above, the efficiency of enhancement of Raman signal by SERS substrates increases significantly, when the frequency of the exciting laser radiation matches or is close to the frequency inherent to the plasmon absorption band of the metal (typically silver, gold). Considering that the wavelengths of laser radiation used in Raman spectroscopy have fixed values, it is more appropriate to adjust the parameters of SERS substrates (shape, size and surface density of nanostructures to the resonance with laser radiation. So, one of the important characteristics of the SERS substrates is their absorption spectra, since the position of the peak and halfwidth of the plasmonic absorption band determine the spectral range in which the significant enhancement of the Raman signal is possible, if the appropriate excitation laser is chosen.

Fig. 4 shows the absorption spectrum of the studied SERS substrates obtained by the first method, namely, by formation of 3 monolayers of NSTs in PDADMAB matrix on a glass substrate. The absorption spectrum of SERS substrates formed by deposition of gold NPs from colloidal solution coincides with the mentioned above. As shown in Fig. 4, the maximum of plasmon absorption band was in $600 \mathrm{~nm}$ region for these substrates. The plasmonic absorption band is quite wide, and its halfwidth approximately equals to $174 \mathrm{~nm}$, which is caused by diversity in the sizes of spherical cores in
NSTs and their edges.

The great halfwidth of the plasmonic absorption band of obtained nanostructuresNS allows to use several lasers with different wavelengths of radiation to excite surface plasmon oscillations. Indeed, as Fig. 4 shows, lasers with the wavelengths of radiation 532 and $632.8 \mathrm{~nm}$ are close enough to the maximum of the plasmon absorption band. Investigations that involved usage of the laser with the wavelength $457 \mathrm{~nm}$ (which does not match with the plasmon absorption band) were performed to estimate the contributions of the electromagnetic and chemical component to the enhancement of the Raman signal.

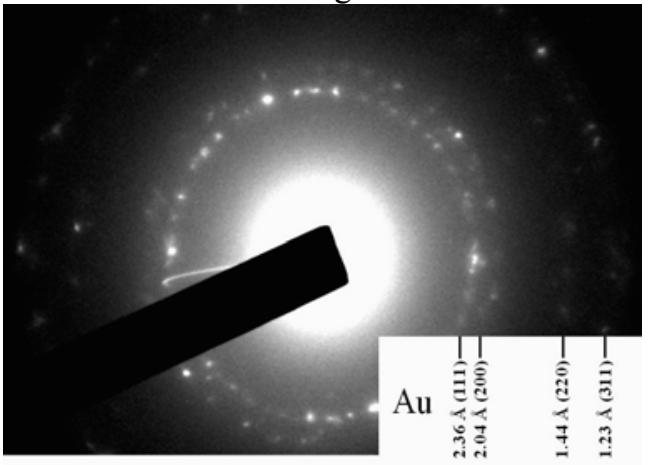

Fig. 2. Picture of electron microdiffraction of the gold nanostars. 


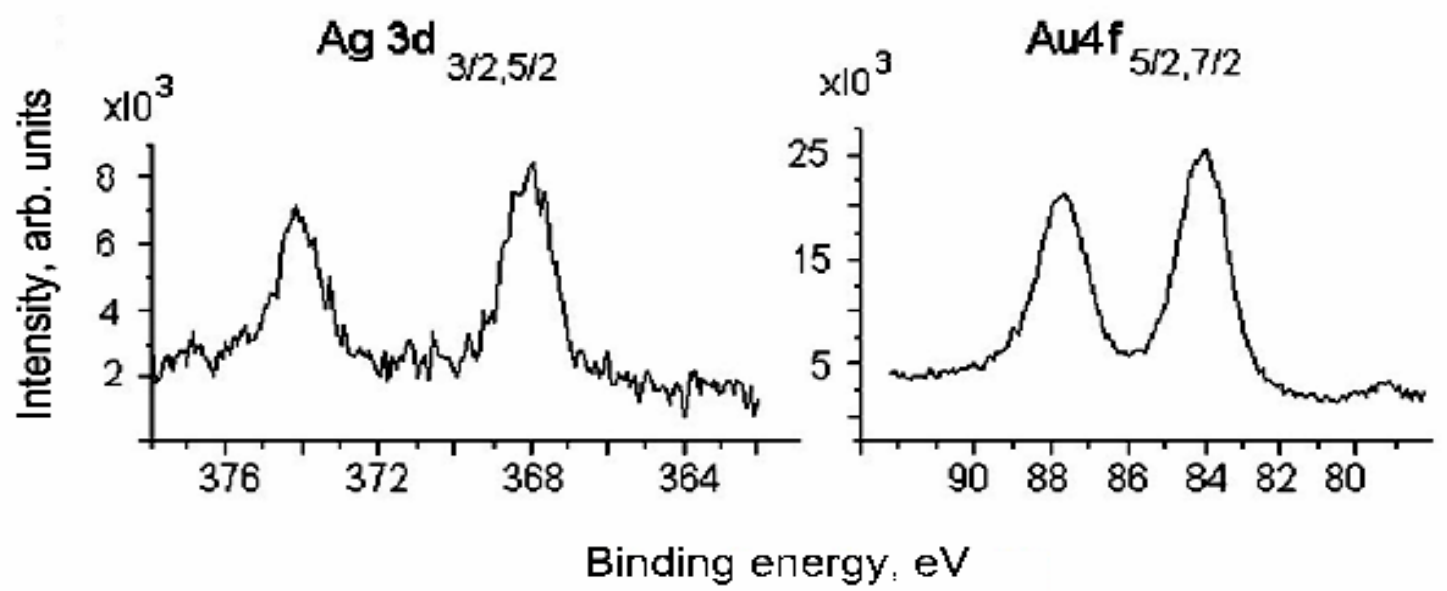

Fig. 3. Photoelectron X-ray spectra of Au4f and $\mathrm{Ag} 3 \mathrm{~d}$ shells from the surface of nanostars.

To correctly estimate the enhancement of the Raman signal by SERS substrates, the same volume of the R6G solution was deposited on substrates, and it was sputtered approximately on the same surface area, the concentration of R6G was of the order of $10^{-3}$ and $10^{-5} \mathrm{M}$. The choice of the concentration of R6G molecules in the solution of the order of $10^{-3} \mathrm{M}$ was caused by the fact that this value was minimal (in this case) sufficient for registration of the Raman signal from R6G molecules deposited on a glass substrate (without gold nanostructures).

The SERS enhancement factor was determined using the formula [21]:

$k=\left(I_{\mathrm{SERS}} / N_{\mathrm{SERS}}\right) /\left(I_{\mathrm{RS}} / N_{\mathrm{RS}}\right)$,

where $I_{\mathrm{SERS}}, I_{\mathrm{RS}}$ are the intensities of SERS and conventional Raman signal, $N_{\mathrm{SERS}}, N_{\mathrm{RS}}$ are the numbers of investigated molecules that contribute to SERS and usual Raman scattering.

In all the cases, calculation of the enhancement factor was carried out using the intensity of the Raman band $\left(612 \mathrm{~cm}^{-1}\right)$ corresponding to R6G molecules, which is caused by $\mathrm{C}-\mathrm{C}$ bending vibrations of the benzene ring [19]. The choice of this Raman band to determine the enhancement factor of SERS substrates was made due to high intensity of it and its far position relatively to the peak of photoluminescence band of R6G molecules, when it was excited by laser radiation.

Experiments have shown that the intensity of $I_{\text {SERS }}$ changes non-linearly with the concentration of the solution of R6G molecules deposited onto the SERS substrate. Indeed, $I_{\text {SERS }}$ depends on the number of investigated molecules that are in the vicinity of $0.1-1 \mathrm{~nm}$ to metallic nanostructures in the place of laser excitation spot. Calculations showed that in this case the solution of R6G molecules with volume of $5 \cdot 10^{-6} 1$ and concentration of $10^{-5} \mathrm{M}$ deposited on substrate cover the area of $3 \cdot 10^{13} \mathrm{~nm}^{2}$, thus forming one monolayer of molecules. Increasing the concentration by one order, it will form a film with the thickness 10 monolayers. But in this case, the main contribution to the intensity of Raman band will be provided by the first layer, which is located directly on metal nanostructures. The contribution of the next layer will be several times smaller and so on; this is caused by exponential decrease of the electric field withdrawing from NP surface. Consequently, it is clear, why in the certain concentration range of solutions further increasing the concentration by one order leads to the increase of $I_{\text {SERS }}$ by $1.5-2$ times.

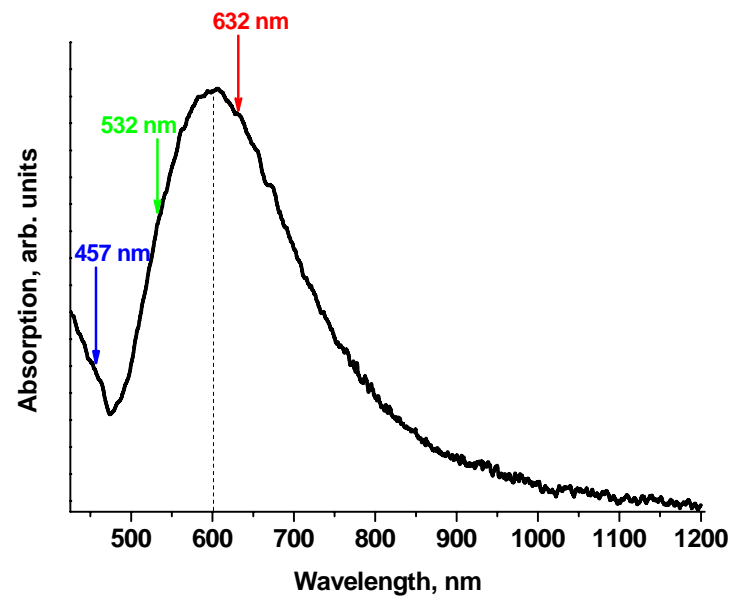

Fig. 4. The absorption spectrum of SERS substrate based on gold nanostars (I type). The arrows show the position of wavelengths for laser excitation used in SERS experiments. 


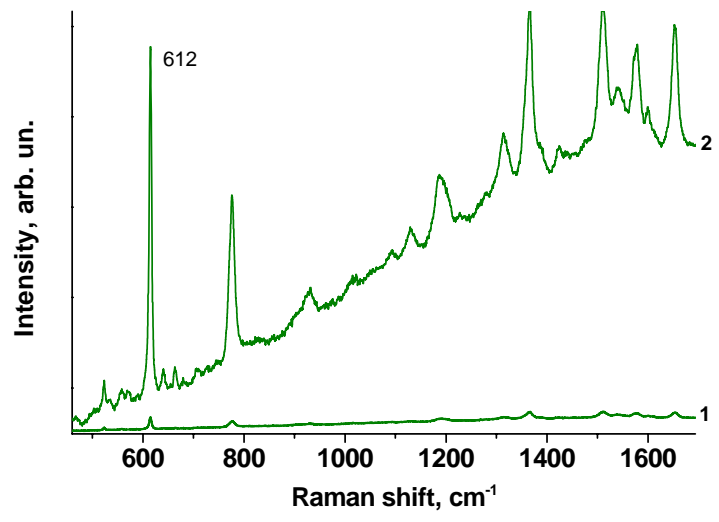

Fig. 5. Raman spectra of R6G molecules deposited on the glass substrate (1) with the concentration of solution $10^{-3} \mathrm{M}$ and on the SERS substrate (II type) (2) with the concentration of the solution $10^{-5} \mathrm{M}$. Spectra were obtained using laser excitation with $\lambda=532 \mathrm{~nm}$.

Fig. 5 shows the Raman spectra of Rhodamine $6 \mathrm{G}$ molecules deposited on the glass (1) and SERS (II type) (2) substrates obtained using excitation with the wavelength $532 \mathrm{~nm}$. It should be noted that R6G molecules were deposited on the glass substrates from the solution with the concentration $10^{-3} \mathrm{M}$, and on the SERS substrate - with the concentration $10^{-5} \mathrm{M}$. Taking into account that the intensity of the bands in the spectrum 2 is higher by 2 orders of magnitude as compared to the spectrum 1 , estimation of the enhancement factor $(k)$ according to the formula (1) gives the value of $10^{4}$. The coefficient of enhancement could be significantly higher in the case when the wavelength of the laser excitation radiation coincidences with the wavelength of the plasmon absorption band peak.

The efficiency of enhancement of the Raman signal of R6G molecules on the SERS substrate (I type), where gold nanostars are placed in the PDADMAB matrix is significantly lower $\left(10^{2}\right.$ times). The coefficient of enhancement of the Raman signal, using the laser excitation radiation with the wavelength $632.8 \mathrm{~nm}$, is 1.5 times higher as compared to that for $\lambda=532 \mathrm{~nm}$. The latter fact is caused by the proximity of the laser light with the wavelength $632.8 \mathrm{~nm}$ to the maximum of plasmon absorption band (602 nm, see. Fig. 2). The decrease of the enhancement by 2 orders of magnitude, as compared to that for the substrate of the first type, is caused by the fact that the gold nanostars are placed in the PDADMAB matrix. On the one hand, it reduces the enhancement due to absence of chemical bonds between the Rhodamine molecules and gold nanoparticles, on the other hand, it reduces the number of hot spots that contribute to enhancement. A partial confirmation of this conclusion could be obtained from the experiment performed with laser radiation of $\lambda=457 \mathrm{~nm}$ for excitation of the Raman spectra for SERS substrates. With this radiation, the intensity of the Raman signal of
R6G molecules was only 2 times higher as compared to the Raman signal from R6G molecules on a glass substrate. The absence of significant enhancement of the Raman signal in SERS substrates of I type can testify about the absence of the chemical bonds between the studied molecules and gold nanostructures.

\section{Conclusions}

The efficient SERS substrates based on films of nanoparticles in the form of stars were developed and investigated. This star-shaped nanostructure enhances the electrical field of radiating dipole not only by setting the frequency of the plasmon absorption band to the frequency of the laser excitation, but also by increasing the number of the so-called hot spots on the surface of substrate. It was shown that these nanostructures efficiently enhance the Raman signal when using laser excitation with the wavelengths 532 and $632.8 \mathrm{~nm}$, which is close to the wavelength of the maximum of plasmon absorption band. For structures that were deposited directly from the colloidal solution, the chemical enhancement is characteristic, because of direct contact of adsorbed molecules with gold nanoparticles. Analysis of the Raman spectra of R6G molecules, deposited on manufactured SERS substrates showed that they enhance Raman signal by 4 orders.

\section{References}

1. Surface-enhanced Raman Scattering Physics and Applications, Eds. K. Kneipp, M. Moskovits, H. Kneipp. Topics in Appl. Phys., Springer-Verlag. 2006. 103. 464 p.

2. Moskovits M. Surface-Enhanced spectroscopy. Rev. Mod. Phys. 1985. 57, No. 3. P. 783-826.

3. Cialla D., März A., Böhme R., Theil F., Weber K., Schmitt M., Popp J. Surface-enhanced Raman spectroscopy (SERS): progress and trends. Anal. Bioanal. Chem. 2012. 403, No. 1. P. $27-54$.

4. Principles of Surface-Enhanced Raman Spectroscopy and Related Plasmonic Effects. Eds. E.C. Le Ru and P.G. Etchegoin. Elsevier, Oxford, UK, 2009. $688 \mathrm{p}$.

5. Quester K., Avalos-Borja M., Vilchis-Nestor A.R., Camacho-Lopez M.A., Castro-Longoria E. SERS properties of different sized and shaped gold nanoparticles biosynthesized under different environmental conditions by neurospora crassa extrac. PLoS One. 2013. 8, No. 10. P. e77486.

6. Chursanova M.V., Germash L.P., Yukhymchuk V.O., Dzhagan V.M., Khodasevich I.A., Cojoc D. Optimization of porous silicon preparation technology for SERS applications. App. Surf. Sci. 2010. 256, No. 11. P. 3369-3373.

7. Sharma B., Frontiera R.R., Henry A.-I., Ringe E., and Van Duyne R.P. SERS: materials, applications, and the future. Materials Today. 2012. 15, No. 1-2. P. 16-25. 
8. Liu T.-Y., Tsai K.-T., Wang H.-H. et al. Functionalized arrays of Raman-enhancing nanoparticles for capture and culture-free analysis of bacteria in human blood. Nature Commun. 2011. 2(538).

9. Tsen M., Sun L. Surface-enhanced Raman scattering from functionalized self-assembled monolayers. Part 1. Distance dependence of enhanced Raman scattering from a terminal phenyl group. Anal. Chim. Acta. 1995. 307, No. 2-3. P. 333-340.

10. Yukhymchuk V.O., Hreshchuk O.M., Valakh M.Ya., Skoryk M.A., Efanov V.S., Matveevskaya N.A. Efficient core- $\mathrm{SiO}_{2} /$ shell-Au nanostructures for surface enhanced Raman scattering. SPQEO. 2014. 1, No. 3. P. 217-221.

11. Zhang W.C., Wu X.L., Kan C.X., Pan F.M., Chen H.T., Zhu J., Chu P.K. Surface-enhanced Raman scattering from silver nanostructures with different morphologies. Appl. Phys. A. 2010. 100, No. 1. P. 83-88.

12. He S., Kang M.W.Ch., Khan F.J., Tan E.K.M., Reyes M.A., Kah J.C.Y., Optimizing gold nanostars as a colloid-based surface-enhanced Raman scattering (SERS) substrate. J. Opt. 2015. 17, No. 11. P. 114013.

13. Fang Y., Seong N.-H., Dlott D.D. Measurement of the distribution of site enhancements in surfaceenhanced Raman scattering. Science. 2008. 321(5887). P. 388-392.

14. Vendrell M., Maiti K.K., Dhaliwal K., Chang Y.-T. Surface-enhanced Raman scattering in cancer detection and imaging. Trends in Biotech. 2013. 31, No. 4. P. 249-257.
15. Qian X., Peng X.-H., Ansari D.O., Yin-Goen Q., Chen G.Z., Shin D.M., Yang L., Young A.N., Wang M.D. \& Nie S. In vivo tumor targeting and spectroscopic detection with surface-enhanced Raman nanoparticle tags. Nature biotech. 2008. 26, No. 1. P. 83-90.

16. Canonico-May S.A., Beavers K.R., Melvin M.J., Alkilany A.M., Duvall C.L., Stone J.W., High conversion of $\mathrm{HAuCl}_{4}$ into gold nanorods: A reseeding approach. J. Col. and Interface Sci. 2016. 463. P. 229-232.

17. Ndokoye P., Li X., Zhao Q., Li T., Tade M.O., Liu S. Gold nanostars: Benzyldimethylammonium chloride-assisted synthesis, plasmon tuning, SERS and catalytic activity. J. Col. and Interface Sci. 2016. 462. P. 341-350.

18. Zhicheng L., Zhaodong Y., Lu B. Layer-by-layer assembly of polyelectrolyte and gold nanoparticle for highly reproducible and stable SERS substrate. Appl. Surf. Sci. 2016. 360(part B). P. 437-441.

19. Richardson J.J., Björnmalm M., Caruso F. Technology-driven layer-by-layer assembly of nanofilms. Science. 2015. 348(6233). P. 411-423.

20. Practical Surface Analysis by Auger and X-ray Photoelectron Spectroscopy, Eds. D. Briggs, M.P. Seach. John Wiley \& Sons Ltd., NY, 1983. 600 p.

21. Le Ru E.C., Blackie E., Meyer M., Etchegoin P.G. Surface enhanced Raman scattering enhancement factors: A comprehensive study. J. Phys. Chem. C. 2007. 111. P. 13794-13803.

22. Hildebrandt P. and Stockburger M. Surfaceenhanced resonance Raman spectroscopy of Rhodamine $6 \mathrm{G}$ adsorbed on colloidal silver. J. Phys. Chem. 1984. 88. P. 5935-5944. 